Mesopotamia J. of Agric.

Vol. (48) No. (1) 2020
ISSN: 2224 - 9796 (Online)

ISSN: 1815 - 316 X (Print)

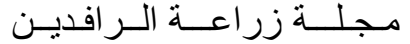

المجلد (48) العدد (1) 2020

\title{
EVALUATION OF FABA BEAN GENOTYPES FOR YIELD AND ITS COMPONENTS USING SELECTION INDEX
}

\author{
M. A. H. Al-Falahy* \\ Kh. H. Kanoosh** \\ *College of Agricultural engineering sciences, Duhok University \\ **Technical Institute in Mosul, Northern Technical University \\ E.mail khalilhathalkn1957@gmail.com
}

\begin{abstract}
The experiment was carried out at the farm of Filed crops Department, College of Agricultural engineering sciences, Duhok University in season 2018-2019 to construed selection index for (Reina, Lattikia, Hadiba, Giza and Aquadocia). under different levels of phosphorus fertilizer(The experiment was laid out in factorial experiment using Randomize Complete Block Design with three replications. The results exhibited that all selection indices include the grain yield gave relatively efficiency, the best selection index were grain yield, number of pods plant ${ }^{-1}$ and number of seeds pods ${ }^{-1}$, so that we can using these characters as criteria's of selection of faba bean genotypes. Also the results showed that the highest mean selection index was 216.26 for lattikia genotype and differs significantly over all other genotypes.

The heritability broad sense was high for all studied characters and ranged between 0.94 to 0.99 . Regarding to the genetic advance as mean gave high values for first pod height (57.10), grain yield plant ${ }^{-1}$ (43.97), number of main branches plant ${ }^{-1}$ (33.92) and number of pods plant ${ }^{-1}$ (31.52) and medium for plant height, days to $75 \%$ flowering, number of nodules plant ${ }^{-1}$ and number of seeds $\operatorname{pod}^{-1}$. So that we can using these desirable characters to improve the faba bean genotypes.
\end{abstract}

Key words: Faba Bean, Production, Selection Index, Phenotypic, Genotypic.

Received: 18/11/ 2019, Accepted: 16 / 1/ 2020

\section{INTRODUCTION}

Genetic improvement mainly depend on the amount of genetic variation existing in the plant population which is a ubiquitous property of feature of all species in nature. The genetic variation found in the germ plasm of a crop, its assessment is pre-requisite for working any effective breeding program, these genetic variability might be either heritable or non-heritable. The estimation of variability due to heritable component is most important because it would be a guide for selection of parents for crop improvement programs of the population. Thus, the genetic variation for agronomic characters is the key component of breeding programs for expression the gene pool of faba bean and others crops. Loos and siddique, (1997) reported that yield improvement is a major breeding objective of most crop improvement programs in faba bean. The success of any plant breeding program depend on the choice of genotypes capable of producing progeny with desired characters combination yield in faba bean, similar to the other crops yield, is complex character and constitute by many of morphological and physiological characters that correlated with others (plant height, pod per plant, 
biological yield, harvest index, 100 -seed weight, days to flowering and maturity) are the most important characters in faba bean improvement for increasing seed yield due to direct and indirect correlation with seed yield.

Arshad et al.,(2001) and sawar et al.,(2004) work on selection index and generally interested improving more than one trait or improving one trait without affecting the performance of outers, and when selection is practical and the correlation of that trait with others is high and undesirable correlated responses may occur for those traits not using in selection criteria. There are three methods have been used to improve several traits but the method using by Hazel and Lush, 1942 is the most efficient method for improving several quantitative characters. selection for superior genotypes depend on the yield a lone was less efficient because the yield and yield components were a complexity characters (Sindhu et al., (1985a) and Sindhu et al., (1985b).Johnson et al., (1955) and Yassin, (1973) reported that the direct selection was in effective due to large environment-genotypes interaction. success in crop breeding program in also depending up on the isolation of genetically superior genotypes based on the amount of variability present in the original population, also the breeder will be able to select the desirable combination of genes. Many desirable characters using from breeder's in faba bean program such as yield, yield components, protein content and quality are under the control of many genes and the environmental factors. Some studies have been carried out on the effectiveness of different selection criteria. approaches for improvement of faba bean characteristics by several investi-gators such as, Nisa and Cengiz, (2011) ; Solieman and Regheb, (2014) ; Yasin and Esrael, (2017) and Kirous (2018).

The objective of this study was to construct several selection indices among eight character of faba bean genotypes and to compare that efficiency with that form the direct selection for grain yield in order to find a simple and useful to be used in the estimation of five faba bean genotypes.

\section{MATERIALS AND METHODS}

The material study consisted of five genotypes of faba bean.The experiment was carried out at the field of College of Agricultural engineering sciences, Duhok University during season 2018-2019.. Grain of five genotypes (Reina, Lattikia, Hadiba, Giza and Aquadocia) were sown in rows, 3 long for each experiment unit, $0.30 \mathrm{~m}$ between rows and $0.10 \mathrm{~m}$ between plants. The date of planting was 27/11/2018. Through seed bed preparation $p_{2} O_{5}$. fertilizer were application at rate of $0,23,46$, and $64 \mathrm{~kg} / \mathrm{ha}$. The data were recorded on a random sample of five guard plants on, plant height $\mathrm{cm}$ first pod height $\mathrm{cm}$, days to $75 \%$ flowering, number of main branches number of pod / plant, number of seeds / pod, number of nodules / plant and wet grain yield per plant.

Analysis of variance of all traits and pairs using procedures describe by Gomez and gomez (1984) and estimate phenotypic and genotypic, covariance of phenotypic and genotypic by using expected mean square from analysis of variance and covariance, Also estimate the following parameter, genetic and phenotypic correlation, 
Broad sense heritability, Expected genetic advance, and genetic advance as mean. The method of constructing the selection indicates were carried out according to Miler etal., (1958) and the comparison between the genotypes means using Duncan's Multiple Range Test (DMRT).

\section{RESULTS AND DISCUSSION}

Table 1. revaluated the analysis of variance for eight characters of faba bean genotypes. It exhibited that the phosphorous fertilizer, faba bean genotypes and their interaction were worthy significant for plant height, first pod height, days to $75 \%$ flowering, number of nodules plant ${ }^{-1}$, main branches plant ${ }^{-1}$,number of pods plant ${ }^{-1}$, number of seeds pod ${ }^{-1}$ and wet grain yield plant ${ }^{-1}$.

The results in Table 2. showed the mean performance of five genotypes at different rates of phosphorus fertilizer for plant height, the tallest plant among all genotypes was produced by Giza at $6.9 \mathrm{~kg} \mathrm{ha}^{-1}$ phosphorous fertilizer with value $130.33 \mathrm{~cm}$, followed by Hudiba at the same rate of phosphate fertilizer, whiles the shortest plant was recorded by Rein a genotype at zero fertilizer. Concerning to first pod height, the Giza and Hudiba were produced the maximum Values (31.66 and 26.66) at rat $69 \mathrm{~kg} \mathrm{ha}^{-1}$ $p_{2} O_{5}$. fertilizer and the Rein a genotype gave the minimum pod height $(10.00 \mathrm{~cm})$ at zero phosphate. For days to $75 \%$ flowering the earliest genotypes was recorded by Rein at $69 \mathrm{~kg} \mathrm{ha}^{-1}$ phosphate with value 84.66 days, while the latest flowering produced by Giza genotype at zero phosphate. Regarding the number of nodule plant ${ }^{-1}$, the Giza genotypes gave the maximum number of nodules with value 97-33 at rate 69 $\mathrm{kg} \mathrm{ha}^{-1} p_{2} o_{5}$., phosphorous $\mathrm{cp} /$ plays important roles in nodulation and biological nitrogen fixation, photosynthesis and nutritional values of Legumes including faba bean, also it has been shown that the induction of plant histidine acid phytase (HAP) gene depends on $\mathrm{p}$ sufficiency in faba bean considerable induction of phytase activities in nodules. These results in agreement with the results proved by Haling et al., (2016); Nasto et al., (2017); and Makoudic et al., (2018). The results in the same table clarified the number of main branches plant ${ }^{-1}$, from this table observed that the Giza genotypes produced the maximum number of branches (6.33) at rate $69 \mathrm{~kg} \mathrm{ha}^{-1} p_{205}$. For the number of pod plant ${ }^{-1}$, the largest number of this trait produced by Lattikia at $46 \mathrm{~kg} \mathrm{ha}^{-1}$ $p_{2} O_{5}$., whereas the smallest number of the main branches remarked by Giza genotype at zero phosphate fertilizer. Similar results were indicated by several authors like Boll et al., (2000); Ragab et al.,(2010) ; Bakty et al.,(2011) and Endalkachew et al., (2018). whose found the addition of phosphate fertilizer was due to an increase in the number of pod plant ${ }^{-1}$ and this trait considerable the major yield components which effected to the final seed yield.

The seeds per pod ${ }^{-1}$ was very considerable among the faba bean genotypes and phosphorous nutrition, for interaction effect of faba bean genotypes are rate of phosphate on seeds per pod $^{-1}$ the same Table exhibited that Lattia genotypes was relatively superior (4.43\%) Reina (3.23), Hudiba (3.26), Giza (3-00) and Aquaduica (3.43) with application at rate $69 \mathrm{~kg} \mathrm{ha}^{-1} p_{2} o_{5}$, this character is a genetic trait and it is in flounced by phosphorous nutrition. From this Table we also notice that the highest 
Mesopotamia J. of Agric.

Vol. (48) No. (1) 2020
ISSN: 2224 - 9796 (Online)

ISSN: 1815 - $316 \mathrm{X}$ (Print)

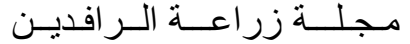
المجلد (48) العدد (1) 2020 الـرن

grain yield (287.96) was produced by Lattikia at $46 \mathrm{~kg} \mathrm{ha}^{-1} p_{2} o_{5}$, while the lowest grain yield $85.5 \mathrm{~g}$ recorded by Rein genotype at Zero $p_{2} o_{5}$.. The increasing grain yield depend on the increase in major components such as number of pods per plant and number of seeds per pod. This traits were un affected by addition of phosphate fertilizer and the kind of genotypes was more over effected in grain yield. The researchers; Lupwayi et al.,(2011) and Endalkachew et al.,(2018), demonstrated similar results for the singe trait

Table (1): Analysis of variance for eight traits of faba bean genotypes

\begin{tabular}{|c|c|c|c|c|c|c|c|c|c|}
\hline \multirow{3}{*}{ S.O.V } & \multirow{3}{*}{ Df } & \multicolumn{8}{|c|}{ Ms } \\
\hline & & \multicolumn{8}{|c|}{ Characters } \\
\hline & & $\begin{array}{c}\text { Plan } \\
\mathrm{t} \\
\text { heig } \\
\mathrm{ht} \\
\mathrm{cm} .\end{array}$ & $\begin{array}{l}\text { First } \\
\text { pod } \\
\text { height } \\
\mathrm{cm} .\end{array}$ & $\begin{array}{c}\text { Days } \\
\text { to } 75 \% \\
\text { floweri } \\
\text { ng }\end{array}$ & $\begin{array}{l}\text { No. of } \\
\text { nodular } \\
\text { plant }^{-1}\end{array}$ & $\begin{array}{c}\text { Main } \\
\text { branches } \\
\text { plant }^{-1}\end{array}$ & $\begin{array}{l}\text { No. of } \\
\text { Pod } \\
\text { plants }^{-1}\end{array}$ & $\begin{array}{c}\text { No. of } \\
\text { Seed } \\
\text { pod }\end{array}$ & $\begin{array}{l}\text { Wet grain } \\
\text { yield }\end{array}$ \\
\hline Replicative & 2 & 13.9 & 6.86 & 0.86 & 22.86 & 0.11 & 0.22 & 0.01 & 60.18 \\
\hline $\begin{array}{c}\text { Phosphorous } \\
\text { A }\end{array}$ & 3 & $\begin{array}{c}* * \\
329 \\
61\end{array}$ & $\begin{array}{c}* * \\
170.0 \\
6\end{array}$ & $\begin{array}{c}* * \\
98.86\end{array}$ & $\begin{array}{c}* * \\
2097.66\end{array}$ & $\begin{array}{c}* * \\
2.46\end{array}$ & 136.22 & $\begin{array}{c}* * \\
1.09\end{array}$ & $\begin{array}{c}* * \\
9382.04\end{array}$ \\
\hline $\begin{array}{c}\text { Genotypes } \\
\text { B }\end{array}$ & 4 & $\begin{array}{c}* * \\
164 \\
9.76\end{array}$ & $\begin{array}{c}* * \\
312.2 \\
0\end{array}$ & $\begin{array}{c}* * \\
455.89\end{array}$ & $\begin{array}{c}* * \\
776.65\end{array}$ & $\begin{array}{c}* * \\
6.87\end{array}$ & 144.15 & $\begin{array}{c}* * \\
1.40\end{array}$ & $\begin{array}{c}* * \\
15207.53\end{array}$ \\
\hline $\mathrm{A} X \mathrm{~B}$ & 12 & $\begin{array}{c}* * \\
13.7 \\
0\end{array}$ & $\begin{array}{c}* * \\
7.63\end{array}$ & $\begin{array}{l}* * \\
7.45\end{array}$ & $\begin{array}{c}* * \\
45.27\end{array}$ & $\begin{array}{c}* * \\
6.69\end{array}$ & 13.311 & $\begin{array}{l}* * \\
0.15\end{array}$ & $\begin{array}{c}* * \\
1604.79\end{array}$ \\
\hline Error & 38 & 4.45 & 1.84 & 2.93 & 3.09 & 0.13 & 0.57 & 0.008 & 5.02 \\
\hline
\end{tabular}


Mesopotamia J. of Agric.

Vol. (48) No. (1) 2020
ISSN: 2224 - 9796 (Online)

ISSN: 1815 - 316 X (Print)

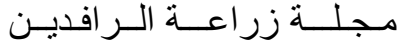

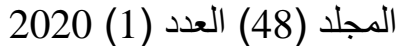

Table (2): Performance of five faba bean genotypes for yield and some agronomic characters at different phosphorus levels.

\begin{tabular}{|c|c|c|c|c|c|c|c|c|}
\hline \multirow{3}{*}{ Combination } & \multicolumn{8}{|c|}{ Mean } \\
\hline & \multicolumn{8}{|c|}{ Characters } \\
\hline & $\begin{array}{l}\mathrm{PH} \\
\mathrm{cm}\end{array}$ & $\begin{array}{l}\text { FPH } \\
\mathrm{Cm}\end{array}$ & 75. fl. & $\begin{array}{l}\text { N. } \\
\text { Nod. }\end{array}$ & M.br. & $\begin{array}{c}\text { N. pod } \\
\text { plant }\end{array}$ & $\begin{array}{c}\mathrm{N} . \\
\text { seed } \\
\text { pod }\end{array}$ & KYP \\
\hline $\mathrm{P}_{0} \mathrm{~V}_{1}$ & $\begin{array}{c}91.66 \\
\text { i }\end{array}$ & $\begin{array}{c}10.00 \\
\mathrm{i}\end{array}$ & $\begin{array}{c}93.66 \\
f\end{array}$ & $\begin{array}{c}52.00 \\
\mathrm{i}\end{array}$ & $\begin{array}{c}3.00 \\
\mathrm{E}\end{array}$ & $\begin{array}{c}17.23 \\
\mathrm{i}\end{array}$ & $\begin{array}{c}3.00 \\
\mathrm{i}\end{array}$ & $\begin{array}{c}126.40 \\
\mathrm{~m}\end{array}$ \\
\hline $\mathrm{P}_{0} \mathrm{~V}_{2}$ & $\begin{array}{c}162.66 \\
\mathrm{fg}\end{array}$ & $\begin{array}{c}13.00 \\
\text { gh }\end{array}$ & $\begin{array}{c}100.66 \\
b c\end{array}$ & $\begin{array}{c}51.33 \\
\mathrm{i}\end{array}$ & $\begin{array}{c}3.33 \\
\text { De }\end{array}$ & $\begin{array}{c}21.96 \\
\text { ef }\end{array}$ & $\begin{array}{c}3.10 \\
\text { ghi }\end{array}$ & $\begin{array}{c}172.86 \\
\mathrm{f}\end{array}$ \\
\hline $\mathrm{P}_{0} \mathrm{~V}_{3}$ & $\begin{array}{c}115.60 \\
\mathrm{~d}\end{array}$ & $\begin{array}{c}17.33 \\
\text { De }\end{array}$ & $\begin{array}{c}103.00 \\
\mathrm{ab}\end{array}$ & $\begin{array}{c}56.33 \\
\mathrm{~h}\end{array}$ & $\begin{array}{c}4.00 \\
\mathrm{Cd}\end{array}$ & $\begin{array}{c}20.23 \\
\text { gh }\end{array}$ & $\begin{array}{c}3.00 \\
\mathrm{i}\end{array}$ & $\begin{array}{c}87.50 \\
h\end{array}$ \\
\hline $\mathrm{P}_{0} \mathrm{~V}_{4}$ & $\begin{array}{c}112.33 \\
\mathrm{~d}\end{array}$ & $\begin{array}{c}18.66 \\
\text { de }\end{array}$ & $\begin{array}{c}104.66 \\
\mathrm{a}\end{array}$ & $\begin{array}{c}62.33 \\
\mathrm{~g}\end{array}$ & $\begin{array}{c}5.00 \\
B\end{array}$ & $\begin{array}{c}16.50 \\
\mathrm{i}\end{array}$ & $\begin{array}{c}2.56 \\
\mathrm{~J}\end{array}$ & $\begin{array}{c}141.53 \\
\mathrm{k}\end{array}$ \\
\hline $\mathrm{P}_{0} \mathrm{~V}_{5}$ & $\begin{array}{c}96.33 \\
\mathrm{~h}\end{array}$ & $\begin{array}{c}11.33 \\
\mathrm{hi}\end{array}$ & $\begin{array}{c}90.33 \\
\text { gh }\end{array}$ & $\begin{array}{c}54.33 \\
\mathrm{hi}\end{array}$ & $\begin{array}{c}5.00 \\
\mathrm{~B}\end{array}$ & $\begin{array}{c}19.20 \\
\mathrm{~h}\end{array}$ & $\begin{array}{c}3.00 \\
\mathrm{I}\end{array}$ & $\begin{array}{c}167.33 \\
\mathrm{~g}\end{array}$ \\
\hline $\mathrm{P}_{1} \mathrm{~V}_{1}$ & $\begin{array}{c}108.00 \\
\mathrm{e}\end{array}$ & $\begin{array}{c}14.33 \\
\text { Fg }\end{array}$ & $\begin{array}{c}97.66 \\
\mathrm{de}\end{array}$ & $\begin{array}{c}70.9 \\
\mathrm{e}\end{array}$ & $\begin{array}{c}4.00 \\
\mathrm{Cd}\end{array}$ & $\begin{array}{c}18.9 \\
\mathrm{i}\end{array}$ & $\begin{array}{c}3.00 \\
\text { I }\end{array}$ & $\begin{array}{c}87.2 \\
n\end{array}$ \\
\hline$P_{1} V_{2}$ & $\begin{array}{c}108.66 \\
\mathrm{e}\end{array}$ & $\begin{array}{c}14.66 \\
\mathrm{fg}\end{array}$ & $\begin{array}{c}97.33 \\
\mathrm{de}\end{array}$ & $\begin{array}{c}70.66 \\
\mathrm{e}\end{array}$ & $\begin{array}{c}4.00 \\
\mathrm{Cd}\end{array}$ & $\begin{array}{c}23.50 \\
\mathrm{~d}\end{array}$ & $\begin{array}{c}3.60 \\
c\end{array}$ & $\begin{array}{c}197.10 \\
\text { d }\end{array}$ \\
\hline$P_{1} V_{3}$ & $\begin{array}{c}118.66 \\
\mathrm{c}\end{array}$ & $\begin{array}{c}119.33 \\
\mathrm{~d}\end{array}$ & $\begin{array}{c}98.66 \\
\mathrm{~cd}\end{array}$ & $\begin{array}{c}78.00 \\
\mathrm{~d}\end{array}$ & $\begin{array}{c}4.00 \\
\mathrm{Cd}\end{array}$ & $\begin{array}{c}20.93 \\
\text { fgh }\end{array}$ & $\begin{array}{c}3.08 \\
\mathrm{hi}\end{array}$ & $\begin{array}{c}162.93 \\
h\end{array}$ \\
\hline$P_{1} V_{4}$ & $\begin{array}{c}108.00 \\
\mathrm{e}\end{array}$ & $\begin{array}{c}17.83 \\
\text { de }\end{array}$ & $\begin{array}{c}94.33 \\
\text { f }\end{array}$ & $\begin{array}{c}72.00 \\
\mathrm{e}\end{array}$ & $\begin{array}{c}4.83 \\
B\end{array}$ & $\begin{array}{c}19.91 \\
\text { gh }\end{array}$ & $\begin{array}{c}2.98 \\
\text { I }\end{array}$ & $\begin{array}{c}146.46 \\
\text { J }\end{array}$ \\
\hline$P_{1} V_{5}$ & $\begin{array}{c}97.00 \\
h\end{array}$ & $\begin{array}{c}13.33 \\
\text { gh }\end{array}$ & $\begin{array}{l}90.66 \\
\text { gh }\end{array}$ & $\begin{array}{c}62.66 \\
\mathrm{~g}\end{array}$ & $\begin{array}{c}4.00 \\
\mathrm{Cd}\end{array}$ & $\begin{array}{c}20.90 \\
\mathrm{fg}\end{array}$ & $\begin{array}{c}3.13 \\
\text { g.i }\end{array}$ & $\begin{array}{c}167.46 \\
\mathrm{~g}\end{array}$ \\
\hline $\mathrm{P}_{2} \mathrm{~V}_{1}$ & $\begin{array}{c}97.00 \\
n\end{array}$ & $\begin{array}{c}14.66 \\
\mathrm{fg}\end{array}$ & $\begin{array}{c}89.00 \\
h\end{array}$ & $\begin{array}{c}67.00 \\
\mathrm{f}\end{array}$ & $\begin{array}{c}4.00 \\
\mathrm{Cd}\end{array}$ & $\begin{array}{c}21.13 \\
\text { gf }\end{array}$ & $\begin{array}{c}3.10 \\
\text { ghi }\end{array}$ & $\begin{array}{c}135.43 \\
\mathrm{~L}\end{array}$ \\
\hline $\mathrm{P}_{2} \mathrm{~V}_{2}$ & $\begin{array}{c}112.33 \\
\mathrm{~d}\end{array}$ & $\begin{array}{c}16.66 \\
\text { ef }\end{array}$ & $\begin{array}{c}95.66 \\
\text { ef }\end{array}$ & $\begin{array}{c}77.66 \\
\mathrm{~d}\end{array}$ & $\begin{array}{c}4.00 \\
\mathrm{Cd}\end{array}$ & $\begin{array}{c}36.60 \\
a\end{array}$ & $\begin{array}{c}4.23 \\
\mathrm{~b}\end{array}$ & $\begin{array}{c}287.96 \\
a\end{array}$ \\
\hline$P_{2} V_{3}$ & $\begin{array}{c}120.66 \\
c\end{array}$ & $\begin{array}{c}23.66 \\
c\end{array}$ & $\begin{array}{c}100.66 \\
\text { bc }\end{array}$ & $\begin{array}{c}86.00 \\
c\end{array}$ & $\begin{array}{c}4.00 \\
\mathrm{Cd}\end{array}$ & $\begin{array}{c}24.86 \\
c\end{array}$ & $\begin{array}{l}3.3 \\
\text { edf }\end{array}$ & $\begin{array}{c}188.63 \\
\mathrm{e}\end{array}$ \\
\hline $\mathrm{P}_{2} \mathrm{~V}_{4}$ & $\begin{array}{c}125.33 \\
b\end{array}$ & $\begin{array}{c}25.66 \\
\mathrm{cb}\end{array}$ & $\begin{array}{c}99.66 \\
\mathrm{~cd}\end{array}$ & $\begin{array}{c}86.33 \\
c\end{array}$ & $\begin{array}{c}6.00 \\
\mathrm{~A}\end{array}$ & $\begin{array}{c}20.66 \\
\text { fg }\end{array}$ & 3.40 & $\begin{array}{c}159.50 \\
h\end{array}$ \\
\hline $\mathrm{P}_{2} \mathrm{~V}_{5}$ & $\begin{array}{c}100.00 \\
\text { gh }\end{array}$ & $\begin{array}{c}14.66 \\
\text { fg }\end{array}$ & $\begin{array}{c}86.00 \\
\text { i }\end{array}$ & $\begin{array}{c}72.66 \\
\mathrm{e}\end{array}$ & $\begin{array}{c}3.33 \\
\mathrm{De}\end{array}$ & $\begin{array}{c}25.40 \\
\mathrm{c}\end{array}$ & $\begin{array}{c}3.40 \\
\mathrm{de}\end{array}$ & $\begin{array}{c}228.23 \\
b\end{array}$ \\
\hline $\mathrm{P}_{3} \mathrm{~V}_{1}$ & $\begin{array}{c}98.66 \\
\mathrm{~h}\end{array}$ & $\begin{array}{c}14.00 \\
\mathrm{~g}\end{array}$ & $\begin{array}{c}84.66 \\
\text { i }\end{array}$ & $\begin{array}{c}70.66 \\
\mathrm{e}\end{array}$ & $\begin{array}{c}4.66 \\
\mathrm{Bc}\end{array}$ & $\begin{array}{c}20.63 \\
f g\end{array}$ & $\begin{array}{c}3.23 \\
F\end{array}$ & $\begin{array}{c}124.63 \\
\mathrm{~m}\end{array}$ \\
\hline $\mathrm{P}_{3} \mathrm{~V}_{2}$ & $\begin{array}{c}113.33 \\
\mathrm{~d}\end{array}$ & $\begin{array}{c}19.33 \\
\mathrm{~d}\end{array}$ & $\begin{array}{c}92.66 \\
\text { fg }\end{array}$ & $\begin{array}{c}78.33 \\
\text { d }\end{array}$ & $\begin{array}{c}4.66 \\
\mathrm{Bc}\end{array}$ & $\begin{array}{c}32.26 \\
b\end{array}$ & $\begin{array}{c}4.43 \\
\mathrm{a}\end{array}$ & $\begin{array}{c}210.26 \\
\mathrm{c}\end{array}$ \\
\hline
\end{tabular}




\begin{tabular}{|c|c|c|c|c|c|c|c|c|}
\hline $\mathrm{P}_{3} \mathrm{~V}_{3}$ & $\begin{array}{c}125.66 \\
\mathrm{~b}\end{array}$ & $\begin{array}{c}26.66 \\
\mathrm{~b}\end{array}$ & $\begin{array}{c}99.00 \\
\mathrm{~cd}\end{array}$ & $\begin{array}{c}92.33 \\
\mathrm{~b}\end{array}$ & $\begin{array}{c}4.66 \\
\mathrm{Bc}\end{array}$ & $\begin{array}{c}25.93 \\
\mathrm{c}\end{array}$ & $\begin{array}{c}3.26 \\
\mathrm{e}-\mathrm{g}\end{array}$ & $\begin{array}{c}139.20 \\
\mathrm{k}\end{array}$ \\
\hline $\mathrm{P}_{3} \mathrm{~V}_{4}$ & $\begin{array}{c}130.33 \\
\mathrm{a}\end{array}$ & $\begin{array}{c}31.66 \\
\mathrm{a}\end{array}$ & $\begin{array}{c}100.66 \\
\mathrm{bc}\end{array}$ & $\begin{array}{c}97.33 \\
\mathrm{a}\end{array}$ & $\begin{array}{c}6.33 \\
\mathrm{~A}\end{array}$ & $\begin{array}{c}21.26 \\
\mathrm{gf}\end{array}$ & $\begin{array}{c}3.00 \\
\mathrm{i}\end{array}$ & $\begin{array}{c}154.96 \\
\mathrm{i}\end{array}$ \\
\hline $\mathrm{P}_{3} \mathrm{~V}_{5}$ & $\begin{array}{c}105.33 \\
\text { ef }\end{array}$ & $\begin{array}{c}18.00 \\
\mathrm{de}\end{array}$ & $\begin{array}{c}85 \\
\mathrm{i}\end{array}$ & $\begin{array}{c}72.33 \\
\mathrm{e}\end{array}$ & $\begin{array}{c}4.66 \\
\mathrm{Bc}\end{array}$ & $\begin{array}{c}23.16 \\
\mathrm{de}\end{array}$ & $\begin{array}{c}3.40 \\
\mathrm{~d}\end{array}$ & $\begin{array}{c}194.66 \\
\mathrm{~d}\end{array}$ \\
\hline
\end{tabular}

Mean with each other column followed by the same letter are not significant at 0.05 and 0.01 probability.

PH: plant height. FPH: first pod height. fl: flowring \%. N.Nod: number of nodes. M.br: main branches. N.seeds/pod: number of seeds/pod.

Table 3 showed that the analysis of variance of five faba bean genotypes and mean of genotypes as a mean to different rates of phosphorous fertilizer. The results in the same table exhibited substantial for all of the studied characters. From this table we also recognized that the Hudiba genotype gave highest plant and latest days to $75 \%$ flowering, while the Giza genotype record the maximum number of nodules plant ${ }^{-1}$ (81.75), maximum of number of main branches plant ${ }^{-1}(5.75)$ and number of pod plant ${ }^{1}$ (19.33) but the Latit kia genotype produced maximum number of seeds $\operatorname{pod}^{-1}$ and wet grain yield plants ${ }^{-1}$. This results were acceptable with Zafar et al., (2011); Agloolik hani et al., (2012) and Tamence et al.,(2015).

Table (3): analysis of variance results for selection index values

\begin{tabular}{|c|c|c|c|c|}
\hline Source & DF & Sum of Squares & Mean Square & F Value \\
\hline Rep & 2 & 29.84526 & 14.92263 & \\
\hline Genotypes & 4 & 15159.13646 & 3789.78411 & $1882.62 * *$ \\
\hline Error & 8 & 16.10428 & 2.01303 & \\
\hline
\end{tabular}

The results in Table 4 referred the estimates of mean square from variance and covariance analysis for estimation of total genetic and phenotypic Variance covariance for the eight Characters, these components used for calculation of genetic and phenotypic correlation, selection indicted, genetic advance that would be excepted from, selection and heritabilities.

The genotypic and phenotypic correlation coefficient among eight characters demons red in Table 5. The quite similar in most cases, and it was exhibited that genotypic correlations exceeded the phenotypic one for majority of cases. The results in the same table remarked that plant height is highly correlated (phenotypically and genotypically) positively with first pod height, days to $75 \%$ flowering and number of nodule plant ${ }^{-1}$ whilst, the first pod height also correlated positively with days to $75 \%$ Flowering number of nodules and main branches plant ${ }^{-1}$. The grain yield per plant is highly correlated with number of pods plant ${ }^{-1}$ and number of seeds pod ${ }^{-1}$ indicating that these two characters are genetically associated with yield, this characters dependent association of this characters and other important ones such as grain yield is general 
very useful to faba bean breeders a similar result were reported by Torres et al., (2006) and Mulualem et al., (2012).

Table 6 Clarified the estimation of genetic, environmental and phenotype variance, heritability broad sense and genetic advance for eight characters of faba bean genotypes. The heritability broad sense was high for all studied characters and ranged between $0.94 \%$ to $99 \%$. Concerning to the genetic advance as mean gave high values for first pod height (57.10), grain yield plant $^{-1}$ (43-97), number of main branches plant ${ }^{-1}$ (33.92) and number of pods plant (31.52) and medium for plant height, days to $75 \%$. flowering, number of nodules plant ${ }^{-1}$ and number of seeds $\operatorname{pod}^{-1}$. For these characters due to their value we can using these characters as criterion of selection, comparable results were obtained by Sindhu et al., (1985a) and Sindhu et al., (1985b); El-Hady et al., (1998) and Solieman et al., (2014).

The data in Table 7. Described the some selection index with relative efficiency over direct for yield for seven of these tested indices. The predicted genetic advance in yield for indices at $10 \%$. Selection intensity score from 36.83 to 80.87 with efficiency 50.28 and 110.39. The best selection index consist from yield, number of pods per plant and number of seeds per pod, there for the better selection indices depend on the yield only to select the better faba bean genotypes. These results were in agreement with Johonson et al., (1955); Sindhu et al.,(1985b); Cerpona et al.(2010) and Kirose, (2018).

Data presented in Table 8. referred to the result analysis of selection indices for faba bean genotypes. The data of all genotypes analyzed using randomized complete block design, the same table was showed from F-test the genotypes mean square was highly significant this main significant differences in selection index means values among genotypes.

The results in the table 9, showed the differences between all genotypes using Duncans Multiple Range - Test and also the same table exhibited that the highest mean selection index was 216.264 for lattikia genotype over all other genotypes, followed by 1 and Giza with values 188.74 and 153.561 significant differences over all other genotypes. Finally it was concluded from this study that the better genotypes lattikia and Giza, the surpassed lattikia and Giza could be used in breeding programs to improve yield at faba bean and develop new hybrid with good productivity and quality performance to Iraqi environments. 
Mesopotamia J. of Agric.

Vol. (48) No. (1) 2020
ISSN: 2224 - 9796 (Online) ISSN: 1815 - 316 X (Print)

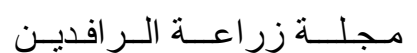
المجلد (48) العدد (1) 2020

Table (4): Genotypic (upper) and phenotypic (lower) variance covariance between studied traits

\begin{tabular}{|c|c|c|c|c|c|c|c|c|}
\hline & grain $\mathrm{yld}$ & $\mathrm{pl} \mathrm{ht}$ & $1 \mathrm{sg}$ pod ht & $75 \%$ flo & nodule pl & main bra & pods pl & seeds pod \\
\hline grain yield gm & $\begin{array}{c}1266.621 \\
1268.643\end{array}$ & -29.5398 & -41.1189 & -13.8639 & -50.952 & -4.41255 & 99.82978 & 10.06485 \\
\hline plant height cm & -29.853 & $\begin{array}{c}136.7365 \\
138.9688\end{array}$ & 57.91458 & 70.36302 & 92.7776 & 5.661979 & 0.430833 & -0.82745 \\
\hline $\begin{array}{c}\text { 1st pod height } \\
\mathrm{cm}\end{array}$ & -40.9523 & 57.32708 & $\begin{array}{c}25.67083 \\
26.71042\end{array}$ & 28.34531 & 40.65781 & 3.016146 & -4.3049 & -0.74344 \\
\hline $75 \%$ flowering & -13.1105 & 70.28854 & 28.81458 & $\begin{array}{l}37.75729 \\
38.45833\end{array}$ & 46.45313 & 2.542188 & 2.285938 & -0.17401 \\
\hline nodules plant & -51.4354 & 93.14583 & 41.27812 & 46.62292 & $\begin{array}{c}64.4125 \\
65.3375\end{array}$ & 4.500521 & -4.41125 & -0.97802 \\
\hline main braches & 4.22906 & 5.639583 & 3.061458 & 2.597917 & 4.498958 & $\begin{array}{c}0.561458 \\
0.595833\end{array}$ & -1.34448 & -0.1438 \\
\hline pods per plant & 100.0335 & 0.244687 & -4.18708 & 2.317812 & -4.38823 & -1.29177 & 11.96841 & 1.141026 \\
\hline seeds per pod & 10.08723 & -0.77979 & -0.76781 & -0.16896 & -0.98469 & -0.14156 & 1.142458 & $\begin{array}{c}0.11625 \\
0.118708\end{array}$ \\
\hline
\end{tabular}


Mesopotamia J. of Agric.

Vol. (48) No. (1) 2020
ISSN: 2224 - 9796 (Online)

ISSN: 1815 - 316 X (Print)

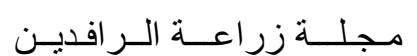

المجلد (48) العدد (1) 2020

Table (5): Genotypic (upper) and phenotypic (lower) correlations between studied traits

\begin{tabular}{|c|c|c|c|c|c|c|c|c|}
\hline & grain yld & pl ht & $\begin{array}{c}\text { 1sg pod } \\
\text { ht }\end{array}$ & $75 \%$ flo & nodule pl & main bra & pods pl & $\begin{array}{c}\text { seeds } \\
\text { pod }\end{array}$ \\
\hline grain yield & 1 & -0.07098 & -0.22803 & -0.0634 & -0.17838 & -0.16547 & 0.810809 & 0.829444 \\
\hline plant height & -0.0711 & 1 & 0.97752 & 0.979269 & 0.988589 & 0.646201 & 0.01065 & -0.20754 \\
\hline 1st pod height & -0.22247 & 0.940938 & 1 & 0.91046 & 0.999859 & 0.794463 & -0.2456 & -0.43036 \\
\hline $\begin{array}{c}75 \% \\
\text { flowering }\end{array}$ & -0.05935 & 0.961459 & 0.899035 & 1 & 0.941953 & 0.552139 & 0.107534 & -0.08306 \\
\hline nodules plant & -0.17865 & 0.977515 & 0.988094 & 0.930086 & 1 & 0.748374 & -0.15888 & -0.13061 \\
\hline main braches & -0.15382 & 0.619764 & 0.767407 & 0.54271 & 0.721055 & 1 & -0.51865 & -0.56287 \\
\hline pods per plant & 0.807317 & 0.005967 & -0.23288 & 0.107436 & -0.15605 & -0.48105 & 1 & 0.967344 \\
\hline seeds per pod & 0.82198 & -0.19199 & -0.4312 & -0.07908 & -0.35357 & -0.53229 & 0.853164 & 1 \\
\hline
\end{tabular}

Table (6): Components of variance, heritability and expected genetic advance for studied traits

\begin{tabular}{|c|c|c|c|c|c|c|c|c|}
\hline & grain yld & $\mathrm{pl} \mathrm{ht}$ & 1sg pod ht & $75 \%$ flo & nodule pl & main bra & pods pl & seeds pod \\
\hline $\mathrm{V} \mathrm{g}$ & 136.7365 & 25.67083 & 37.75729 & 64.4125 & 0.561458 & 11.96841 & 0.11625 & 1266.621 \\
\hline $\mathrm{V} \mathrm{e}$ & 2.232292 & 1.039583 & 0.701042 & 0.925 & 0.034375 & 0.133781 & 0.002458 & 2.02165 \\
\hline $\mathrm{V} \mathrm{p}$ & 138.9688 & 26.71042 & 38.45833 & 65.3375 & 0.595833 & 12.10219 & 0.118708 & 1268.643 \\
\hline heritability & 0.983937 & 0.961079 & 0.981771 & 0.985843 & 0.942308 & 0.988946 & 0.979291 & 0.998406 \\
\hline GA & 23.89423 & 10.23215 & 12.54217 & 16.41558 & 1.498381 & 7.08715 & 0.695056 & 73.25618 \\
\hline GA\% & 21.85114 & 57.10966 & 13.19997 & 22.92145 & 33.92561 & 31.52412 & 21.29895 & 43.87168 \\
\hline
\end{tabular}

Vg: genetic variance, ve: environment variance, vp: phenotypic variance, h.b.s: heritability broad sense, GA: genetic advance, GA\%: genetic advance as mean. 
Mesopotamia J. of Agric.

Vol. (48)
ISSN: 2224 - 9796 (Online) ISSN: 1815 - $316 \mathrm{X}$ (Print)

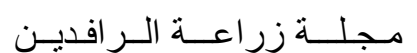
المجلد (48) العدد (1) 2020

Table (7): Some selection indices with their relative efficiency.

\begin{tabular}{|c|c|c|c|c|c|c|c|c|c|}
\hline grain yld & plant ht & $\begin{array}{c}\text { 1st pod } \\
\text { height }\end{array}$ & $\begin{array}{c}75 \% \\
\text { flowering }\end{array}$ & $\begin{array}{c}\text { nodule } \\
\text { per plant }\end{array}$ & $\begin{array}{c}\text { No main } \\
\text { bran }\end{array}$ & $\begin{array}{c}\text { pods per } \\
\text { plant }\end{array}$ & $\begin{array}{c}\text { seeds per } \\
\text { pod }\end{array}$ & GA & RE\% \\
\hline 0.9984 & & & & & & & & 73.25594 & 100 \\
\hline 0.999235 & & & & & & -0.01051 & & 79.84925 & 109.0004 \\
\hline 0.999708 & & & & & & & -0.1637 & 79.84927 & 109.0004 \\
\hline 0.999625 & & & & & & 0.01154 & -0.26772 & 80.84921 & 110.3654 \\
\hline & & & & & & 2.6779 & 59.0139 & 65.8978 & 89.95557 \\
\hline & & -16.0463 & 4.5929 & 4.7744 & 18.9665 & & & 36.83928 & 50.28845 \\
\hline 1.01369 & & & & & -0.74585 & 0.0216 & -2.44922 & 80.85413 & 110.3721 \\
\hline 0.999272 & & & & 0.007629 & & -0.02259 & 0.153979 & 79.84932 & 109.0005 \\
\hline 1.00105 & & -0.80462 & -0.44432 & 0.90867 & -1.09008 & & & 79.88411 & 109.048 \\
\hline 1.05168 & & & & 0.28615 & -3.46211 & -1.22174 & 5.42278 & 80.8741 & 110.3994 \\
\hline 1.04212 & 0.12585 & & & & -2.51368 & -0.82247 & 1.97669 & 79.86599 & 109.0232 \\
\hline 1.04318 & & 0.40867 & & & -3.15497 & -1.08554 & 5.47082 & 79.86572 & 109.0229 \\
\hline 1.01176 & -0.66247 & -1.30418 & -0.35318 & 2.29966 & -2.64478 & -0.75417 & 8.70195 & 79.90601 & 109.0779 \\
\hline
\end{tabular}


Mesopotamia J. of Agric.

Vol. (48) No. (1) 2020
ISSN: 2224 - 9796 (Online)

ISSN: 1815 - $316 \mathrm{X}$ (Print)

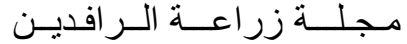
المجلد (48) العدد (1) 2020 الرند

Table (8): Analysis of variance results for selection index values

\begin{tabular}{|c|c|c|c|c|}
\hline Source & DF & Sum of Squares & Mean Square & F Value \\
\hline rep & 2 & 29.84526 & 14.92263 & \\
\hline genotypes & 4 & 15159.13646 & 3789.78411 & $1882.62 * *$ \\
\hline Error & 8 & 16.10428 & 2.01303 & \\
\hline
\end{tabular}

Table (9): means of selection index values for the five genotype

\begin{tabular}{|c|c|c|}
\hline genotypes & $\begin{array}{c}\text { Means of selection index } \\
\text { values }\end{array}$ & $\begin{array}{c}\text { Sequence of genotypes according to their } \\
\text { preference }\end{array}$ \\
\hline 1 & $129.011 \mathrm{e}$ & 5 \\
\hline 2 & $216.264 \mathrm{a}$ & 1 \\
\hline 3 & $143.931 \mathrm{~d}$ & 4 \\
\hline 4 & $153.561 \mathrm{c}$ & 3 \\
\hline 5 & $188.740 \mathrm{~b}$ & 2 \\
\hline
\end{tabular}

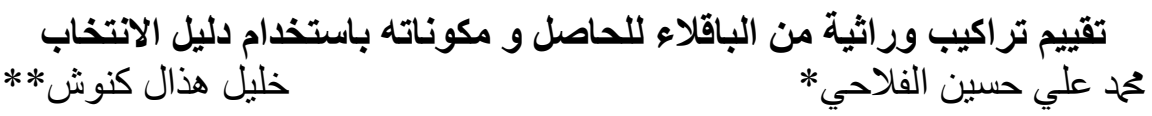

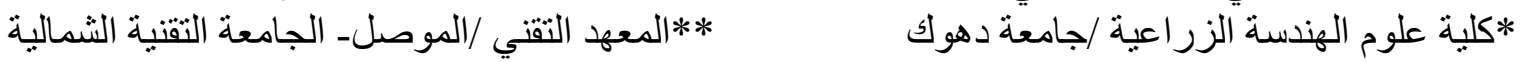

E.mail khalilhathalkn1957@gmail.com

\section{الخلاصة}

طبقت الدر اسة في حقل قسم المحاصيل الحقلية التابع الى كلية علوم الهندسة الزر اعية / جامعة دهوك خلاصل

الموسم 2018-2019 لانثـاء ادلة انتخاب في (Reina, Lattikia, Hadiba, Giza and Aquadocia)

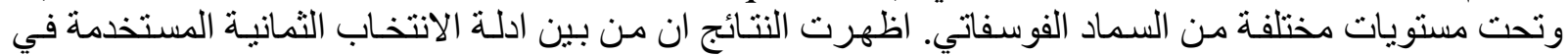

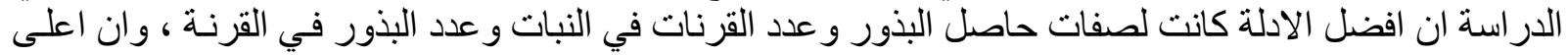

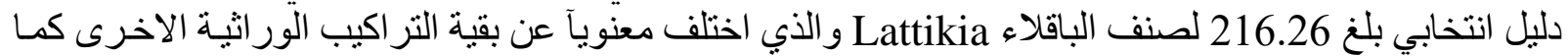

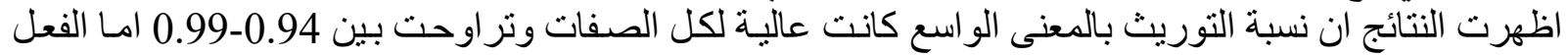

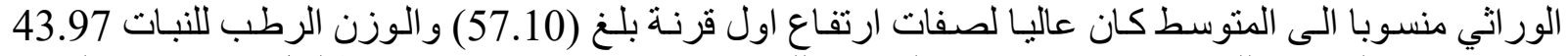
و عدد الافر ع الرئيسية للنبات (33-92) و عدد القرنات للنبات

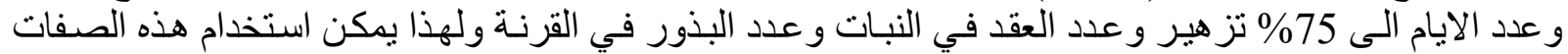
المرغوبة في تحسين هذه التر اكيب الور الوانية.

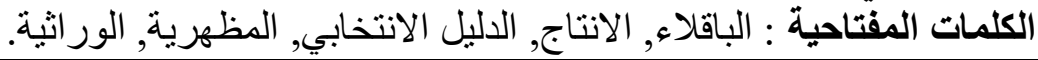

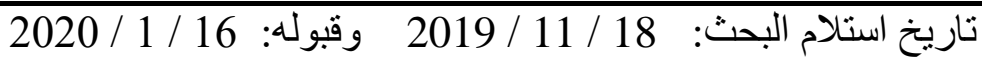

\section{REFERENCES}

Agloolik hani M, Faw. Etemadi and A. F. Agir (2012). Phsiological basis of yield difference for grain Sorgum insemi arid environment. Agric.bio. sci.7.PP.

Arshd. M,A. Bakhsh and A. Ghafoor.(2001). path coefficient analysis chick pea (Cicer aritinum) under field condition. Paka. J. Bot, 36.1:75-81.

Bakry. B-A,T.T. AElewa, M.F.E, Karamanr, M. S. Zedian and M.M. Taufik. (2011). Effect of row spacing on yield and it components of some faba bean varieties 
Mesopotamia J. of Agric.

Vol. (48) No. (1) 2020
ISSN: 2224 - 9796 (Online)

ISSN: 1815 - 316 X (Print)
مـلــــة زر اعــة الــر افديـن

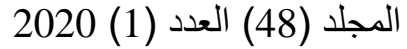

under newly - Re-claimed sand soil condition. world Journal of Agri. Sci.7(1): 68-72

Boll, M.D.A, K.H.M. Siddiave and R. F. Brennau. (2000). Grain yield response of faba beam (vicia faba 1) to application of fertilizer phosphorus and zinc, Australian Journal of experimental Agriculture. $40: 849-857$.

Crepona K, Marget. P. Peyronnet. C, carrone ea, B, Arese P, Duc G. (2010). Nutritional value of faba bean (vicia faba 1.) seeds for feed and food. Field crops Ros, 115: 329-339.

El-Hady M.M, Gad El-Karim GA, Abou-zeid NM (1998) inheritance of resistance to Chocolate spot disease Botrytis faba and heterosis in faba bean (vicia faba 1.) Egyptian J. Genet. and cytol. 27 (1):1-9.

Endalkachew. F, k.G, kibert, A. Melese. A.M. Bobe.(2018). yield of faba bean (vica faba L.) as affed by time minerals, farm yard manure compost and rhizobium in acid soil of lay gayint district, north western high lands of Ethiopa. Agriculture and food security. 7-16

Fouad,M, Hu. Jinguo, M. Donal, O. Sullivan, 2. Xuxiao, H. Aladdin, k. shiv and B. Michael..(2018). Breeding and genomics status in faba bean (vicia faba). plant breeding Journal 29.:1-9.

Gomez, k. A and A.A. Gomez. (1984). Statistical Procedures for Agricultural Research, $2^{\text {nd }}$ Edition. John Wiley and stone. New York.

Haling. R. E, Yang, IV. shadwell and R.J. Simpson (2016). Growth and root dry matter allocation by pasture Legumes and grass with contrasting external critical phosphorous requirement. plant soil J. 407:67-79.

Hazel, L.N, and J.L. Lush. (1942). The efficiency of three methods of selection. $J$. Hered, 33:393-399

Johanson, H. W., H. P. Robinson and R.E cosmtock. (1955). Genetic and enjro nmeutal variability in soya bean. Agro. J. 47: 314-318

Kiros wol day.(2018). Evaluation of faba bean (V.F.L.) varieties for yield and yield related traits in Central zone of Tigray Northern. Ethiopia Journal of plant breeding and crop science 9:258.261

Loss. s. p, and K-H. M. Siddique. (1997). adaptation et faba bean (Vicia faba L) to dry land Mediterranean -type environment. 1.seed yield and yield components. Field Crops Research. G52: 17-28.

Lupwayi. N. Z., Kennedy. A.c and R. Mchiwa. (2011). Grain Legumes impact on soil biological processes in sub - Sahran Africa. Africa Journal of plant sciencesas.5:1-7

Makoudie. B.A.K. Kabbadi, M. Mouradi, L. Amenc, O. Domergue, M. Blai and C. Ghoulam. (2018). Phosphorus deficiency increase nodule phytase activity of faba bean-rhizobia symbiosisiacta J. physiol. plant. V.40, no.3, pp: 63-67.

Miller, P.A, J.C. Williams, H. F. Robinson and R.E. coumstock. (1958). Estimation genotypic and environmental variance and covariance in selection. Argon. J. Voliso pp: 126-131. 
Mulualem. T, Dessalegn. T, Dessalegn V.. (2012). participatory Varietal Selection of faba been (v. f. 1) for yield and yield components in Dabat district, Ethiopia, wodpecker J. Agric. Res.l 270-274.

Nisa. E.l and Cengiz. F. (2011). Screening and Selection of faba bean (V.F.L.) for cold tolerance and comparison to wild relatives. Springer science 58: 1169-1175.

Nosto. N.K, B.B. Osborne, Y. lekberg, G.P. Asshe, C.S Baliotti, S. porderand and c.c. (level and. (2017). Nutrient acquisitions, soil Phosphorous partitioning and competition among tress in a lowland and tropical rain forest. New phyto,214:1506-1517.

Ragab, A.A,A. E Man, A, Tantawy and sh. M. Abd-El-Rasoul (2010). A comparison between traditional and recent bio inocula on grow and productivity of faba bean (vicia faba L.) grown in calcareous soil. International. J. Academic Res., 2(4): 245-253.

Sawar. G, M. s. sadig, M. Seleem and G. Abbas. (2004). Selection criteria in F3 and F4 population of mang bean (vigna.rdaiata L) wilczek. Pak.J. Bot. 36,2:297-310.

Sindhu. J.S, O.p. singh and K.p. singh (1985a) Component Analysis of The Factors Cletermining Grain Yield in Faba Been (V. F. L.) FABIS News Letter ICARDA.

Sindhu. J.S, O.P. Singh and K.P. Singh (1985b) Selection Indices in Faba Bean (V. F. L.) FABIS. 12. 7. In Selection Indices and Genetic Advance in Faba Bean (V. F. L.) FABIS News Letter ICARDA.

Solieman. T. H.l and E-1.m. Ragheb. (2014). Two selection methods and Estimation of some important Genetic parameters in bread bean (V. F. L.) Asian Journal of crop science. $6(1): 38-48$.

Tamence. T,k. Gemechu, s.taclese and J. Mussa. (2015). Yield stability and relationships among stability parameters in faba bean (vicia faba L.) genotypes. Crop Journal. 3, 4: 258-268.

Torres. A.M, Roman. B, Avila. cM, Satovic. 2, Rubiales. D, sillero. J.c, Cubero J.1, Moreno. M.T. (2006). Faba Bean breeding for resistance against biotic stresses: to words application of marker technology. Euphytica :147: 67- 80.

Yasin. G. and k. Esrael. (2017). participatory on Farm Evaluation and Selection of improved Faba Bean (V. F. L.) varieties in for District of South Ethiopia. Advance in crop science and Technology. 5. 293- 30l.

Yassin, T.A. (1973). Genotypic and phenotypic in faba bean (V.F.L.) in the Northern province of the Sudan. s. of Agric. Sai. Cambridge 80:119-129.

Zafar. M. Abbasi, M. K Rakin, N. khalig, A, shaheen, A. Jamil. M and Shahid. (2011). Influence of integrated phosphorous supply and plant growth promoting rhizo bacteria on growth nodulation, yield and nutrient uptake in phaselous vulgaris. friva jour. of Bio. 10.6793-16807. 